\title{
Interobserver agreement in perineal ultrasound measurement of the anovaginal distance: a methodological study
}

\author{
Sofia Pihl ${ }^{1}$ - Eva Uustal ${ }^{1,2} \cdot$ Linda Hjertberg ${ }^{1,2} \cdot$ Marie Blomberg $^{1,2}$
}

Received: 10 February 2017 / Accepted: 29 April 2017 / Published online: 17 June 2017

(C) The Author(s) 2017. This article is an open access publication

\begin{abstract}
Introduction and hypothesis Objective outcome measures of the extent of laceration at delivery are needed. In this study we evaluated and describe here a method for learning perineal ultrasound measurement of the anovaginal distance (AVD). The learning period needed for examiners proficient in vaginal ultrasound examination and the interobserver agreement after reaching proficiency in AVD measurement were determined. The hypothesis was that the method is feasible to learn and reproducible for use in further research.

Methods The method was taught by an examiner experienced in perineal ultrasonography. The distance between the mucosal margin of the internal anal sphincter was measured with a vaginal probe. The studied examiners measured the AVD until similar results $( \pm 5 \mathrm{~mm})$ were achieved. The AVD in 40 women was then measured and documented by two examiners who were blinded to each other's results. Interobserver agreement was calculated using the kappa score.

Results Examiners with previous experience in vaginal ultrasonography had learned the method after performing five sets of comeasurements. The AVD measurements after the learning period showed almost perfect agreement $(\kappa=0.87)$ between the examiners.

Conclusions The method for perineal ultrasound measurement of AVD was learned quickly with high interobserver
\end{abstract}

Eva Uustal

eva.uustal@regionostergotland.se

Marie Blomberg

marie.blomberg@regionostergotland.se

1 Department of Obstetrics and Gynaecology, Linköping University, SE-581 85 Linköping, Sweden

2 Department of Clinical and Experimental Medicine, Linköping University, Linköping, Sweden agreement. The method is feasible to learn and reproducible for use in further research.

Keywords Perineal ultrasound examination - Anovaginal distance $\cdot$ Perineum $\cdot$ Interobserver agreement $\cdot$ Validation

\section{Introduction}

Perineal lacerations after delivery are common and need careful evaluation and treatment to avoid significant morbidity. Current methods for pelvic floor evaluation after delivery are, however, subjective and vary in quality, and lacerations are often misdiagnosed and undertreated [1]. Objective, accessible and reproducible clinical examination methods for identifying the extent of lacerations could increase patient safety and facilitate comparative research [2].

The perineal tissue between the anal mucosa and the vaginal wall at the middle level of the anal canal consists of the anal mucosa, internal anal sphincter, external anal sphincter and the fibrous tissue that is the insertion point for the external sphincter, transverse perineal, bulbocavernosus and puborectalis muscles, as well as the rectovaginal fascia. In previous research, daily practice and reconstructive surgery, this tissue is referred to as the perineal body [3-5]. In more recent studies the perineal body is described in more detail using histopathology and various imaging modalities [6,7]. Ultrasound can be used for evaluating the anatomy of the female pelvic floor [8-11]. Both endoanal and endovaginal 3-D ultrasonography require specific skills and equipment, today only found at specialized centres. In contrast, equipment for vaginal/perineal ultrasonography is available in all labour wards in Sweden and obstetricians/ gynaecologists use it in daily practice. The perineal approach has been shown to be accurate for imaging the anal sphincters and the perineal tissue [12]. 
With a vaginal probe directed backwards from the distal vagina toward the anus, the anovaginal distance (AVD) can be measured [13]. The examination is quick and painless for the woman. If the AVD is short, some or all the perineal components are missing or thin [3]. As the AVD is a new concept, the relationship between the AVD and the perineal body is unknown. Whether or not the AVD could serve as a proxy indicator for obstetric perineal damage depends on the reliability of the measurement method. It has to be both easy to learn and reproducible to be generally useful in delivery wards. This study was designed to evaluate the use of perineal ultrasonography for measuring the AVD, in terms of both ease of learning by the examiner and interobserver agreement.

\section{Materials and methods}

The study population was recruited from among women attending the gynaecological outpatient clinic of a university hospital between May 2013 and June 2014. Exclusion criteria were inability to understand spoken or written information. Printed study information was posted in the waiting-room. Examination of the study subjects included vaginal ultrasonography and had to be performed when two examiners were on duty on the ward at the same time. There was no selection regarding patient characteristics since the study objective was merely to evaluate the measurement technique. All women who were given written and verbal information about the study gave consent. Three examiners were chosen based on their different levels of experience of vaginal ultrasonography, which were 5, 15 and 21 years. As do most Swedish gynaecologists, they used vaginal ultrasonography in daily clinical practice to measure internal genitalia distances.

First, a pilot study was conducted to establish if the method was feasible to learn. We stipulated that five sets of comeasurements would be sufficient to learn the method. Five consenting women were measured each three times by the two examiners, one with and one without previous experience of AVD measurement. The technique was openly discussed and adjusted according to the instructions for measurement presented below. When the examiners produced three consecutive similar AVD values $( \pm 5 \mathrm{~mm})$, they were considered proficient (data not shown). This was achieved after performing five sets of three measurements, and the method was considered robust enough for study of interobserver agreement.

All examinations were done with the woman in the lithotomy position as is standard in Swedish gynaecological practice. The equipment was a Bk Medical Flex Focus 5001202 ultrasound scanner with a type 8819 9-5 MHz vaginal probe. The study was approved by the Ethics Review Board of Linköping University Hospital.

The measurement instructions were as follows:
1. Place the vaginal probe at right angles to the posterior distal vaginal wall in a transverse scanning plane (see Fig. 1).

2. Move the probe slowly cranially from the distal anal canal to the point where the internal anal sphincter first appears as a low-echogenic ring.

3. Adjust the image size so that the internal sphincter ring fills more than half the screen.

4. Steady the probe against the tissue using light pressure until the image begins to be distorted, then release the pressure until an undistorted image is just restored.

5. Freeze the image and measure the distance between the anal limit of the internal anal sphincter and the edge of the probe; this represents the AVD.

6. Repeat twice and record the measurements.

Figure 2 shows the point of measurement defined as the midanal canal [14].

The first examiner made the first three measurements and silently documented them in the study protocol. The second examiner was then called in and made three measurements and documented them on a separate page of the protocol. The examiners did not see or hear each other's results. In two women in whom the internal sphincter was torn and the limit could not be defined, the distance between the outer edge of the anal mucosa and the vaginal probe was measured. The mean AVD based on the three measurements obtained by each examiner were paired for every patient. Interobserver agreement was assessed using the weighted kappa coefficient and a Bland-Altman plot. Table 1 shows the definitions used to interpret the kappa coefficient $[15,16]$.

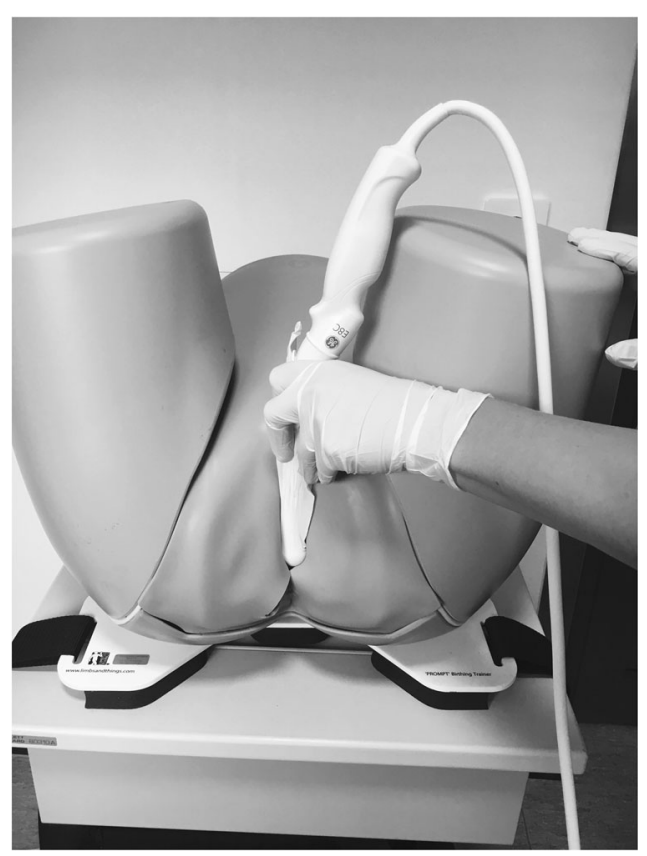

Fig. 1 Technique for measuring the anovaginal distance 


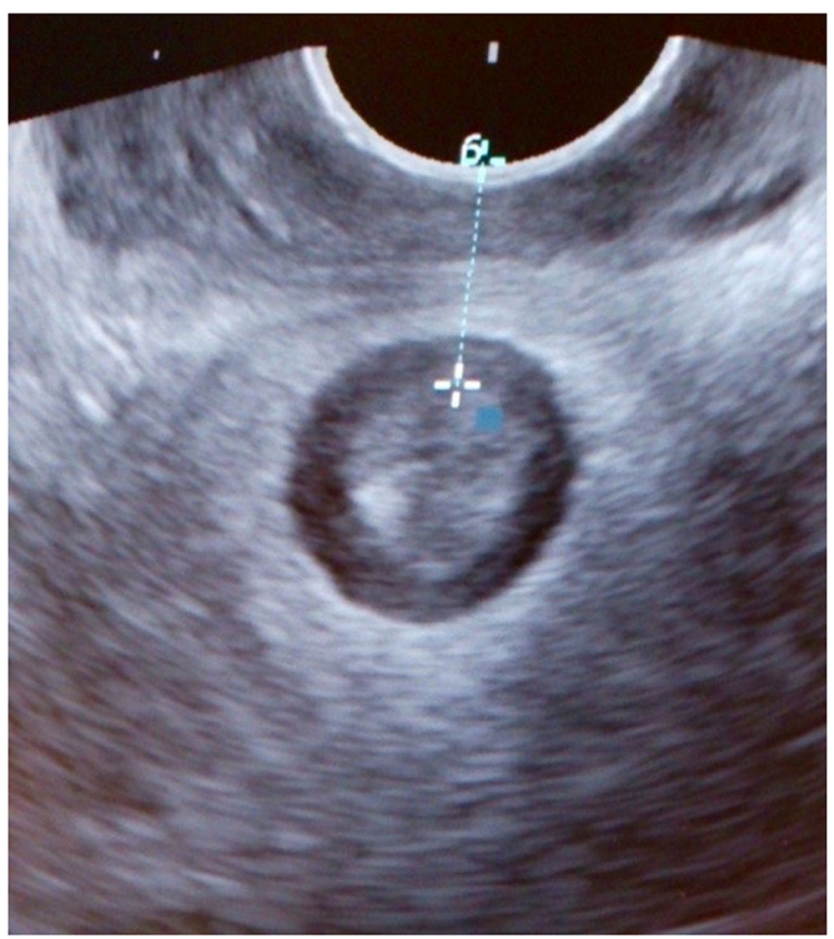

Fig. 2 Perineal ultrasound image, obtained with a vaginal probe showing measurement of the anovaginal distance

Power calculations were based on data from a pilot study of the use of perineal ultrasonography for the measurement of AVD in five women performed by two examiners. The main study needed 40 women to have a power of $80 \%$ at a twosided significance level of alpha $=0.05$ with an accepted difference of $5 \mathrm{~mm}$ between examiners. The value of $5 \mathrm{~mm}$ was based on clinical experience. Forty women were recruited.

The statistical analysis was performed using Stata v. 13.1 (Statacorp LP, College Station, TX).

\section{Results}

A new examiner achieved proficiency after comeasuring five patients each three times. Three measurements were then made by both examiners in all 40 women. No discomfort was reported by the women. The mean difference in the AVD measurements was $1.8 \mathrm{~mm}$ (95\% CI 1.13-2.45 mm, $99 \%$ CI $0.91-2.68 \mathrm{~mm}$ ). There was one outlier of a difference of $10.1 \mathrm{~mm}$, and three values more than the accepted difference of $\leq 5 \mathrm{~mm}$ between the examiners, all of which were included in the calculations. With an accepted difference of $\leq 5 \mathrm{~mm}$ interobserver variation, the weighted kappa coefficient was $0.87(p \leq 0.001)$ with an agreement of $92.5 \%$, classified as almost perfect agreement (Table 1). Variation in the measurements was not significantly influenced by the length of the AVD. Interobserver variation is shown in Fig. 3.
Table 1 Definitions of the levels of agreement in relation to the kappa coefficient $(k)$

\begin{tabular}{ll}
\hline Kappa coefficient & Level of agreement \\
\hline$\leq 0.20$ & Slight \\
$0.21-0.40$ & Fair \\
$0.41-0.6$ & Moderate \\
$0.61-0.80$ & Substantial \\
$0.81-1.00$ & Almost perfect \\
\hline
\end{tabular}

The intraobserver variability ranged from 0.1 to $3.9 \mathrm{~mm}$ and there was no difference in variability in relation to years of experience. Examining systematic errors comparing mean measurements and standard deviations, there were no significant differences between individual examiners regardless of their experience with the use of vaginal ultrasonography in basic gynaecological clinical care (data not shown).

The mean age of the participants was 47 years (range 1883 years) and their mean parity was 2 (range $0-5$ ). Demographic data of the study population are presented in Table 2.

\section{Discussion}

Perineal ultrasonography has a short learning period among examiners routinely performing vaginal ultrasound examinations. The method of measuring AVD evaluated in the present study seems to work and can be recommended for implementation in clinical practice. This is the first study of a method for teaching AVD measurement and the interobserver agreement among doctors with proficiency in vaginal ultrasonography. Interobserver agreement with the use of endovaginal 3-D ultrasonography of the pelvic floor has been shown to be good [17]. In studies of endoanal ultrasonography, a distance of less than $10 \mathrm{~mm}$ between the anal mucosa and the vaginal wall measured at the mid-anal canal level has been shown to be

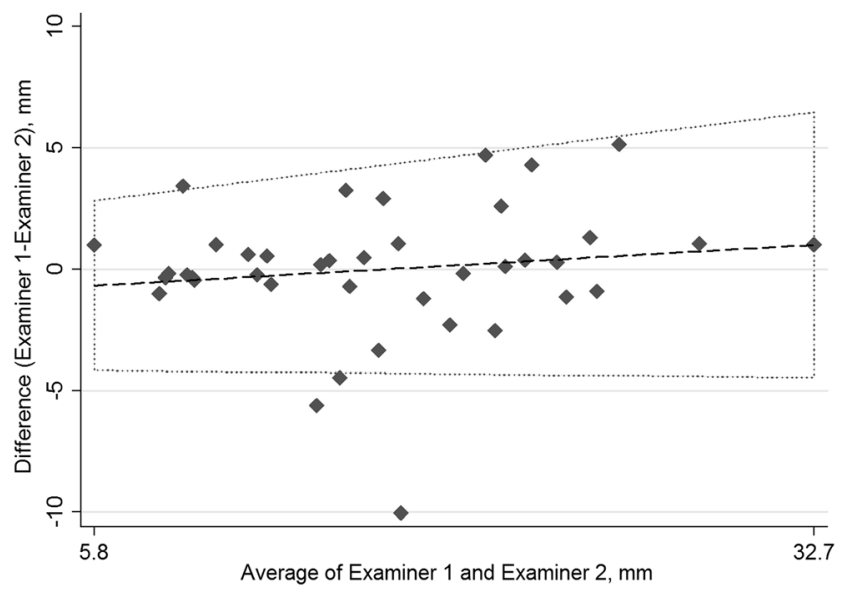

Fig. 3 Bland-Altman plot of interobserver differences in measured anovaginal distance 
Table 2 Demographic data of the study population

Study protocol no. Year of birth Age (years) Parity Caesarean section Anovaginal Reason for visit Additional medical information distance $(\mathrm{mm})$

\begin{tabular}{|c|c|c|c|c|c|c|c|}
\hline 1 & 1959 & 55 & 2 & 0 & 8 & Lichen planus & Urinary incontinence, MP \\
\hline 2 & 1961 & 53 & 3 & 0 & 10 & Rectocele & Faecal incontinence, TVT, MP \\
\hline 3 & 1959 & 55 & 2 & 0 & 21 & Urinary urgency & TVT, MP, ERT \\
\hline 4 & 1931 & 83 & 2 & 0 & 9 & Cystocele & MP, local ERT \\
\hline 7 & 1973 & 41 & 4 & 0 & 28 & Cervical dysplasia & \\
\hline 8 & 1935 & 79 & 5 & 0 & 19 & Cystocele & MP, local ERT \\
\hline 9 & 1961 & 53 & 2 & & 23 & Intrauterine device & MP \\
\hline 10 & 1988 & 26 & 0 & 0 & 17 & Cervical dysplasia & \\
\hline 11 & 1975 & 39 & 2 & 0 & 25 & Cervical dysplasia & \\
\hline 12 & 1974 & 40 & 1 & 0 & 20 & Cervical dysplasia & \\
\hline 13 & 1952 & 62 & 1 & 0 & 18 & Cervical dysplasia & MP \\
\hline 14 & 1938 & 76 & 2 & 0 & 16 & Urinary incontinence & TAH + SOE, MP \\
\hline 15 & 1974 & 40 & 2 & 0 & 20 & Urinary incontinence & \\
\hline 16 & 1979 & 35 & 3 & 0 & 33 & Cervical dysplasia & \\
\hline 17 & 1967 & 47 & 2 & 0 & 25 & Urinary incontinence & \\
\hline 18 & 1960 & 54 & 3 & 0 & 17 & Cervical dysplasia & \\
\hline 19 & 1988 & 26 & 1 & 1 & 15 & Urinary urgency & \\
\hline 20 & 1988 & 26 & 0 & 0 & 15 & Cervical dysplasia & \\
\hline 21 & 1996 & 18 & 2 & 0 & 14 & Cervical dysplasia & \\
\hline 22 & 1940 & 74 & 3 & 0 & 15 & Urinary incontinence & Rectocele, cystocele, MP \\
\hline 23 & 1970 & 44 & 3 & 0 & 9 & Bleeding & Myoma \\
\hline 24 & 1953 & 61 & 2 & 0 & 10 & Pelvic pain & TAH, rectocele. MP \\
\hline 25 & 1972 & 42 & 3 & 1 & 12 & Abdominal pain & \\
\hline 26 & 1957 & 57 & 0 & 0 & 12 & Cervical dysplasia & MP \\
\hline 27 & 1980 & 34 & 2 & 0 & 12 & Faecal incontinence & \\
\hline 28 & 1979 & 35 & 3 & 0 & 14 & Pelvic pain & \\
\hline 29 & 1959 & 55 & 0 & 0 & 17 & Urinary urgency & MP \\
\hline 30 & 1964 & 50 & 4 & 0 & 10 & Rectocele & TVT, MP \\
\hline 31 & 1953 & 61 & 3 & 0 & 9 & Rectocele & MP \\
\hline 32 & 1968 & 46 & 2 & 0 & 9 & Cystocele & Rectocele, TVT \\
\hline 33 & 1995 & 19 & 0 & 0 & 16 & Labia minora surgery & \\
\hline 34 & 1985 & 29 & 0 & 0 & 12 & Miscarriage & \\
\hline 35 & 1989 & 25 & 1 & 0 & 6 & Rectocele & \\
\hline 36 & 1975 & 39 & 3 & 0 & 22 & Cervical dysplasia & \\
\hline 37 & 1963 & 51 & 2 & 1 & 21 & Abdominal pain & \\
\hline 38 & 1992 & 22 & 0 & 0 & 15 & Cervical dysplasia & \\
\hline 39 & 1965 & 49 & 3 & 1 & 22 & Dysmenorrhea & \\
\hline 40 & 1953 & 61 & 3 & 0 & 21 & Bleeding & \\
\hline 41 & 1951 & 63 & 2 & 0 & 24 & Urinary incontinence & Rectocele, cystocele \\
\hline 42 & 1970 & 44 & 2 & 0 & 23 & Intrauterine device & \\
\hline
\end{tabular}

TVT tension-free vaginal tape, MP Menopausal, ERT Estrogen replacement therapy, TAH Total abdominal hysterectomy, SOE Salpingooophorectomy

correlated with anal sphincter injury and anal incontinence [3, $18,19]$. Transperineal ultrasonography performed with a vaginal probe has been used to detect occult sphincter injuries directly after delivery [9]. Measurements of the perineal tissue components using this approach in the immediate postpartum period have not yet been reported.

The strengths of this study include the following, First, all measurements were made live in the clinical examination situation and not on images or video recordings analysed retrospectively, which indicates that the interobserver validity found is transferable to other clinical settings. Second, the women examined were not selected from a population with pelvic floor dysfunction, so that the examiners would have had no expectations of any ultrasound findings in the perineal area.
The study also had some limitations. The subjects were examined as gynaecological outpatients and not directly after delivery. For establishing reproducibility, it was considered more ethically sound to test the method in a calm setting. Also, all examiners were motivated to make the method work. How the teaching protocol will work among general staff in the delivery ward is now the subject of further studies.

There is an urgent need to establish objective outcome measures regarding perineal lacerations to evaluate preventative interventions and risk factors [2]. Even though postpartum endoanal ultrasonography of the perineal area shows missed lacerations and prevents anal incontinence [1], it is rarely used. The use of perineal ultrasonography for the measurement of AVD is less hindered by a lack of equipment and skills than endoanal ultrasonography. Having established its 
feasibility and reproducibility, we are now going on to evaluate perineal ultrasonography in clinical studies for the examination of women after delivery.

\section{Conclusions}

Perineal ultrasound measurement of the AVD showed a short learning period for examiners with previous experience in ultrasound examination as well as a high interobserver agreement. The method described can be taught and reliably used in further research.

\section{Compliance with ethical standards}

\section{Conflicts of interest None.}

Open Access This article is distributed under the terms of the Creative Commons Attribution 4.0 International License (http:// creativecommons.org/licenses/by/4.0/), which permits unrestricted use, distribution, and reproduction in any medium, provided you give appropriate credit to the original author(s) and the source, provide a link to the Creative Commons license, and indicate if changes were made.

\section{References}

1. Faltin DL, Boulvain M, Floris LA, Irion O. Diagnosis of anal sphincter tears to prevent fecal incontinence: a randomized controlled trial. Obstet Gynecol. 2005;106(1):6-13.

2. Jiang H, Qian X, Carroli G, Garner P. Selective versus routine use of episiotomy for vaginal birth. Cochrane Database Syst Rev. 2017;2:CD000081.

3. Fornell EU, Matthiesen L, Sjödahl R, Berg G. Obstetric anal sphincter injury ten years after: subjective and objective long term effects. BJOG. 2005;112(3):312-6.

4. Shobeiri SA, Nolan TE, Yordan-Jovet R, Echols KT, Chesson RR. Digital examination compared to trans-perineal ultrasound for the evaluation of anal sphincter repair. Int J Gynaecol Obstet. 2002;78(1):31-6.

5. Larson KA, Yousuf A, Lewicky-Gaupp C, Fenner DE, DeLancey JO. Perineal body anatomy in living women: 3-dimensional analysis using thin-slice magnetic resonance imaging. Am J Obstet Gynecol. 2010;203(5):494.e15-21.

6. Stein TA, DeLancey JO. Structure of the perineal membrane in females: gross and microscopic anatomy. Obstet Gynecol. 2008;111(3):686-93.
7. Petros PE. Vault prolapse I: dynamic supports of the vagina. Int Urogynecol J Pelvic Floor Dysfunct. 2001;12(5):292-5.

8. Starck M, Bohe M, Fortling B, Valentin L. Endosonography of the anal sphincter in women of different ages and parity. Ultrasound Obstet Gynecol. 2005;25(2):169-76.

9. Ozyurt S, Aksoy H, Gedikbasi A, Yildirim G, Aksoy U, Acmaz G, et al. Screening occult anal sphincter injuries in primigravid women after vaginal delivery with transperineal use of vaginal probe: a prospective, randomized controlled trial. Arch Gynecol Obstet. 2015;292(4):853-9.

10. Ros C, Martínez-Franco E, Wozniak MM, Cassado J, Santoro GA, Elías N, et al. Postpartum two- and three-dimensional ultrasound evaluation of anal sphincter complex in women with obstetric anal sphincter injury. Ultrasound Obstet Gynecol. 2017;49(4):508-14.

11. Oberwalder M, Thaler K, Baig MK, Dinnewitzer A, Efron J, Weiss EG, et al. Anal ultrasound and endosonographic measurement of perineal body thickness: a new evaluation for fecal incontinence in females. Surg Endosc. 2004;18(4):650-4.

12. Timor-Tritsch IE, Monteagudo A, Smilen SW, Porges RF, Avizova E. Simple ultrasound evaluation of the anal sphincter in female patients using a transvaginal transducer. Ultrasound Obstet Gynecol. 2005;25(2):177-83.

13. Frudinger A, Bartram CI, Kamm MA. Transvaginal versus anal endosonography for detecting damage to the anal sphincter. AJR Am J Roentgenol. 1997;168(6):1435-8.

14. Hall RJ, Rogers RG, Saiz L, Qualls C. Translabial ultrasound assessment of the anal sphincter complex: normal measurements of the internal and external anal sphincters at the proximal, mid-, and distal levels. Int Urogynecol J Pelvic Floor Dysfunct. 2007;18(8): 881-8.

15. Viera AJ, Garrett JM. Understanding interobserver agreement: the kappa statistic. Fam Med. 2005;37(5):360-3.

16. Cerro CR, Franco EM, Santoro GA, Palau MJ, Wieczorek P, EspuñaPons M. Residual defects after repair of obstetric anal sphincter injuries and pelvic floor muscle strength are related to anal incontinence symptoms. Int Urogynecol J. 2017;28(3):455-60.

17. Santoro GA, Wieczorek AP, Shobeiri SA, Mueller ER, Pilat J, Stankiewicz A, et al. Interobserver and interdisciplinary reproducibility of 3D endovaginal ultrasound assessment of pelvic floor anatomy. Int Urogynecol J. 2011;22(1):53-9.

18. Uustal Fornell E, Wingren G, Kjølhede P. Factors associated with pelvic floor dysfunction with emphasis on urinary and fecal incontinence and genital prolapse: an epidemiological study. Acta Obstet Gynecol Scand. 2004;83(4):383-9.

19. Zetterström JP, Mellgren A, Madoff RD, Kim DG, Wong WD. Perineal body measurement improves evaluation of anterior sphincter lesions during endoanal ultrasonography. Dis Colon Rectum. 1998;41(6):705-13. 